INPLASY

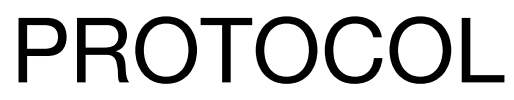

To cite: Wu et al. Association between serum lipids and breast cancer risk in premenopausal women: systematic review and metaanalysis. Inplasy protocol 202190011. doi: 10.37766/inplasy2021.9.0011

Received: 05 September 2021

Published: 05 September 2021

Corresponding author:

Wei Li

sejishikong508@163.com

Author Affiliation:

Chongqing Key Laboratory of Translational Research for Cancer Metastasis and Individualized Treatment, Chongqing University Cancer Hospital.

Support: KJQN201800109, 2021 MSXM222.

Review Stage at time of this submission: Data analysis Completed but not published.

Conflicts of interest:

None declared.

\section{Association between serum lipids and breast cancer risk in premenopausal women: systematic review and meta- analysis}

\author{
Wu, X1; Li, W22; Zeng, X33; Lei, X; Pan, X5.
}

Review question / Objective: Analyze whether dyslipidemia is a high risk factor for breast cancer in premenopausal women by comparing the difference in blood lipids between premenopausal women with breast cancer and healthy premenopausal women.

Condition being studied: To our knowledge, no previous metaanalysis has specifically evaluated the role of dyslipidemia in breast cancer risk in premenopausal women.

Information sources: We searched literatures on PubMed, Embase, China National Knowledge Infrastructure, and Wanfang data without restrictions until 31 December 2020.

INPLASY registration number: This protocol was registered with the International Platform of Registered Systematic Review and Meta-Analysis Protocols (INPLASY) on 05 September 2021 and was last updated on 05 September 2021 (registration number INPLASY202190011).

\section{INTRODUCTION}

Review question / Objective: Analyze whether dyslipidemia is a high risk factor for breast cancer in premenopausal women by comparing the difference in blood lipids between premenopausal women with breast cancer and healthy premenopausal women.

Condition being studied: To our knowledge, no previous meta-analysis has specifically 
evaluated the role of dyslipidemia in breast cancer risk in premenopausal women.

\section{METHODS}

Participant or population: Premenopausal breast cancer patients.

Intervention: Premenopausal women newly diagnosed with breast cancer.

Comparator: Healthy premenopausal women with normal physical examination.

Study designs to be included: Eligible studies were identified by searching the literatures in PubMed, Embase, China National Knowledge Infrastructure, and Wanfang data until December 31, 2020. The standardized mean difference (SMD) score with $95 \%$ confidence interval $(\mathrm{CI})$ was used to assess the impact of serum lipids on premenopausal breast cancer risk. The Isquared statistic was calculated to measure the percentage of heterogeneity, and the Egger tests were performed to measure publication bias.

Eligibility criteria: 1) all subjects in the case group were histopathologically diagnosed as having primary breast cancer before menopause and did not start any antitumor treatment; and 2) the risk factors explored included at least one of the selected lipid components (TG, TC, LDL-C, andHDL-C).

Information sources: We searched literatures on PubMed, Embase, China National Knowledge Infrastructure, and Wanfang data without restrictions until 31 December 2020.

Main outcome(s): Thirteen studies were included. The SMD scores of triglycerides (TG) and low-density lipoprotein cholesterol (LDL-C) were 12.90 (95\% Cl: 7.19-18.61) and 31.43 (95\% Cl: $8.72-54.15)$ respectively. However, the SMD score of total cholesterol (TC) was 31.10 (95\% Cl: $-9.98-$ 70.18), and that of high-density lipoprotein cholesterol (HDL-C) was -1.39 (95\% Cl: -2.90-0.12) without statistical difference. The studies involved in our meta-analysis were highly heterogeneous. There was no publication bias in the TC, LDL-C, and HDL-C analyses, whereas publication bias was present in the TG analysis.

Quality assessment / Risk of bias analysis: Publication bias was tested using Egger's test considering the small study effect. No evidence of publication bias was found in the TC $(P=0.189)$, LDL-C $(p=0.107)$, and HDL-C $(p=0.575)$ analyses, whereas evidence of publication bias was found in the TG $(p=0.001)$ analysis.

Strategy of data synthesis: We used STATA version 15.1 (STATA Corp., College Station, TX) for all analyses.The I-squared (I2) statistic was calculated to measure the percentage of heterogeneity. When substantial heterogeneity was detected, the random effect models were used. Otherwise, fixed effect models were used to assess the effect size of the included studies in the meta-analysis. Egger tests were performed to measure the publication bias. A p-value of less than 0.05 was considered statistically significant.

Subgroup analysis: Subgroup analyses were equally performed based on different races for African and Asian populations.

Sensitivity analysis: None.

Country(ies) involved: China.

Keywords: premenopausal breast cancer, serum lipids, meta-analysis.

Contributions of each author:

Author 1 - Jing Wu.

Author 2 - Wei Li.

Author 3 - Xiaohua Zeng.

Author 4 - Xun Lei.

Author 5 - Xianjun Pan. 